\title{
Patterns in the Juan Fernandez fur seal faecal microbiome
}

\author{
Constanza Toro Valdivieso ${ }^{1}$, Frederick Toro ${ }^{2}$, Samuel Stubbs ${ }^{3}$, Eduardo Castro-Nallar ${ }^{2}$, \\ and Barbara Blacklaws ${ }^{1}$ \\ ${ }^{1}$ University of Cambridge \\ ${ }^{2}$ Universidad Andres Bello Departamento de Ciencias Biologicas \\ ${ }^{3}$ London School of Hygiene and Tropical Medicine Faculty of Epidemiology and Public \\ Health
}

May 6, 2021

\begin{abstract}
As apex predators, pinnipeds are considered to be useful bioindicators of marine and coastal environments. Endemic to a small archipelago in the South Pacific, the Juan Fernandez fur seal (JFFS) is one of the less-studied members of the pinniped family Otariidae. This study aimed to characterize the fecal microbiome of the JFFS for the first time, in order to establish a baseline for future studies of host-microbial-environment interactions and monitoring programs. During two consecutive reproductive seasons, 57 fecal samples were collected from 7 different JFFS colonies within the Juan Fernandez Archipelago, Chile. Bacterial composition and abundance were characterized by sequencing the V4 region of the $16 \mathrm{~S}$ rRNA gene. The overall microbiome composition was dominated by five phyla: Firmicutes (40\%), Fusobacteria (30\%), Bacteroidetes (22 \%), Proteobacteria (6 \%) and Actinobacteria (2\%). Alpha diversity was higher in Tierras Blancas. However, location was not found to be a dominant driver of microbial composition. Interestingly, the strongest signal in the data was a negative association between the genera Peptoclostridium and Fusobacterium, which explained $29.7 \%$ of the total microbial composition variability between samples. The genus Peptoclostridium has not been reported in other pinniped studies and its role here is unclear, with interpretation challenging due to a lack of information regarding microbiome functionality in marine mammals. As a first insight into the JFFS fecal microbiome, these results contribute towards our understanding of the natural microbial diversity and composition in free-ranging pinnipeds.
\end{abstract}

\section{Hosted file}

Microbial_patterns_2021.pdf available at https://authorea.com/users/412267/articles/521014patterns-in-the-juan-fernandez-fur-seal-faecal-microbiome 

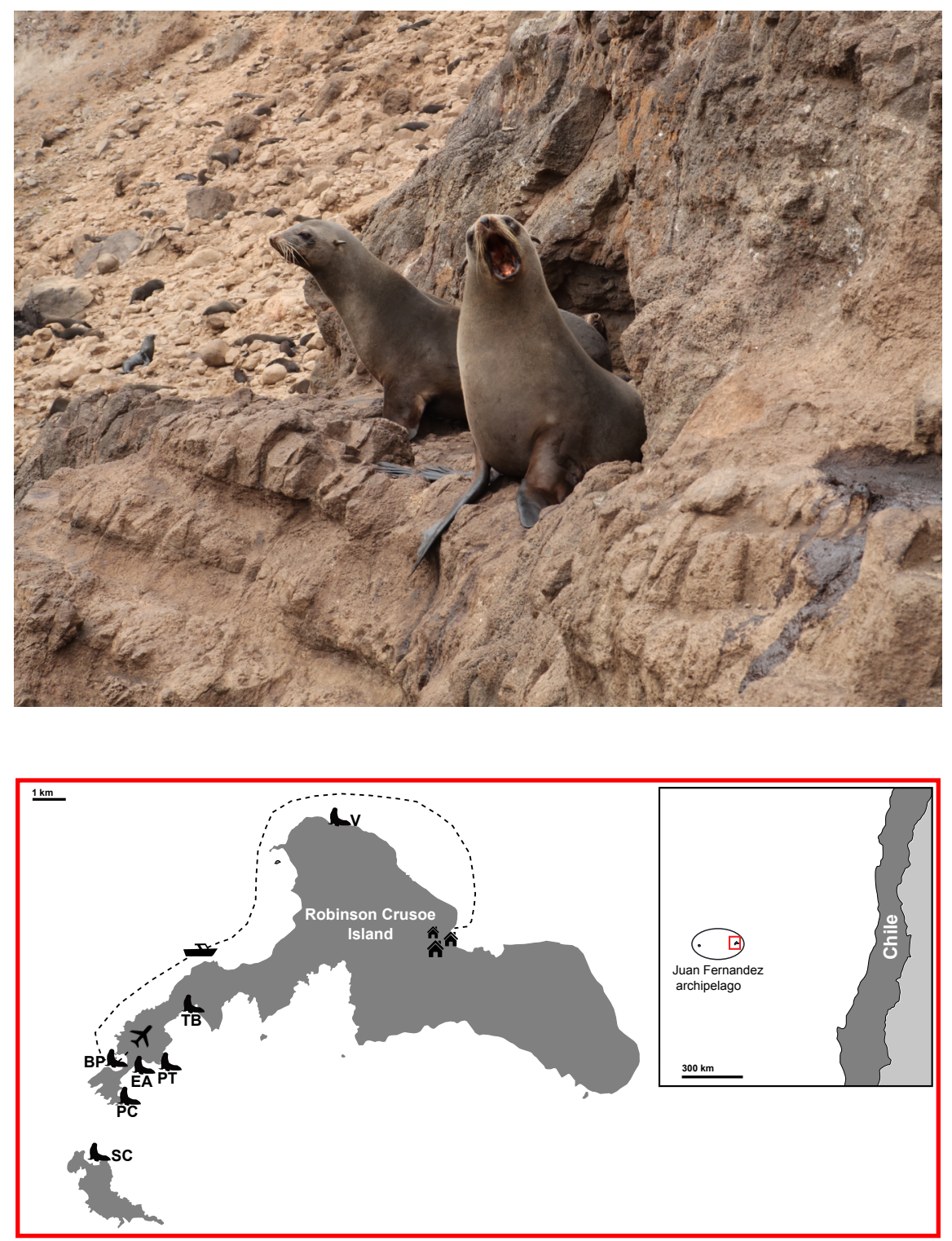
3.A

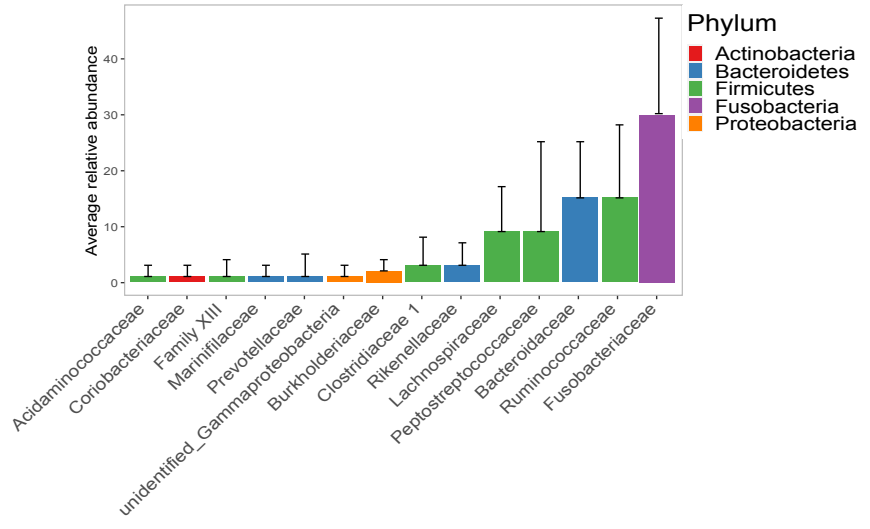

3.B

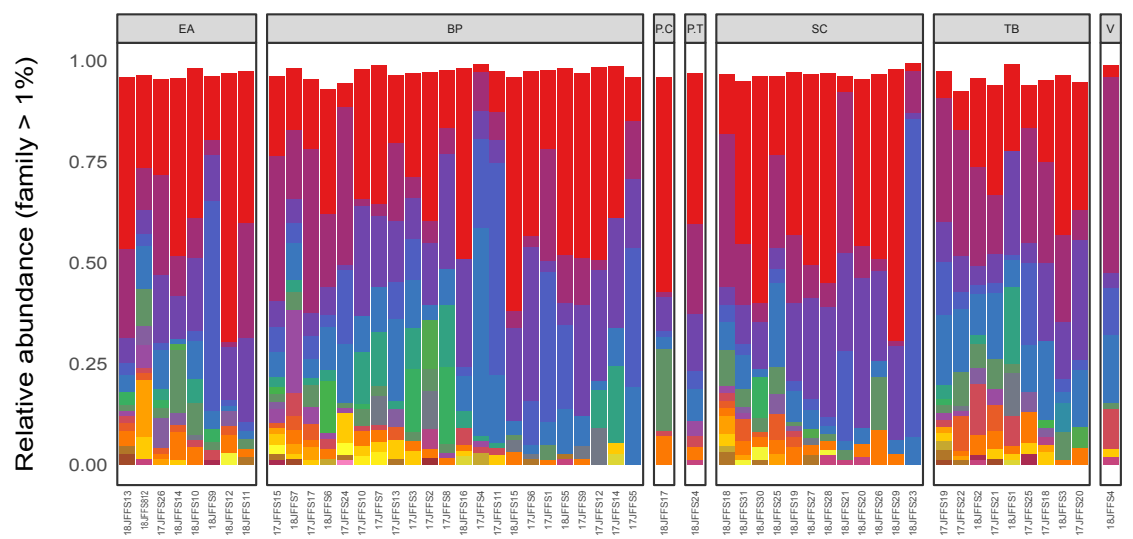

- Fusobacteriaceae

Ruminococcacea

Peptostreptococ

Pasteurellaceae

Clostridiaceae 1

Prevotellaceae

Akkermansiaceae

Succinivibrionace

Rikenellaceae

naproteobacteria

Family XIII vadinBB60 group

Helicobacteraceae
Coriobacteriaceae

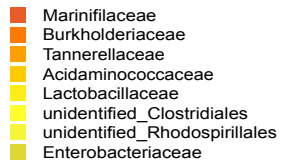

Eggerthellaceae

Disionaceae

unidentified_Bacteria

Erysipelotrichacea

Eubacteriaceae 

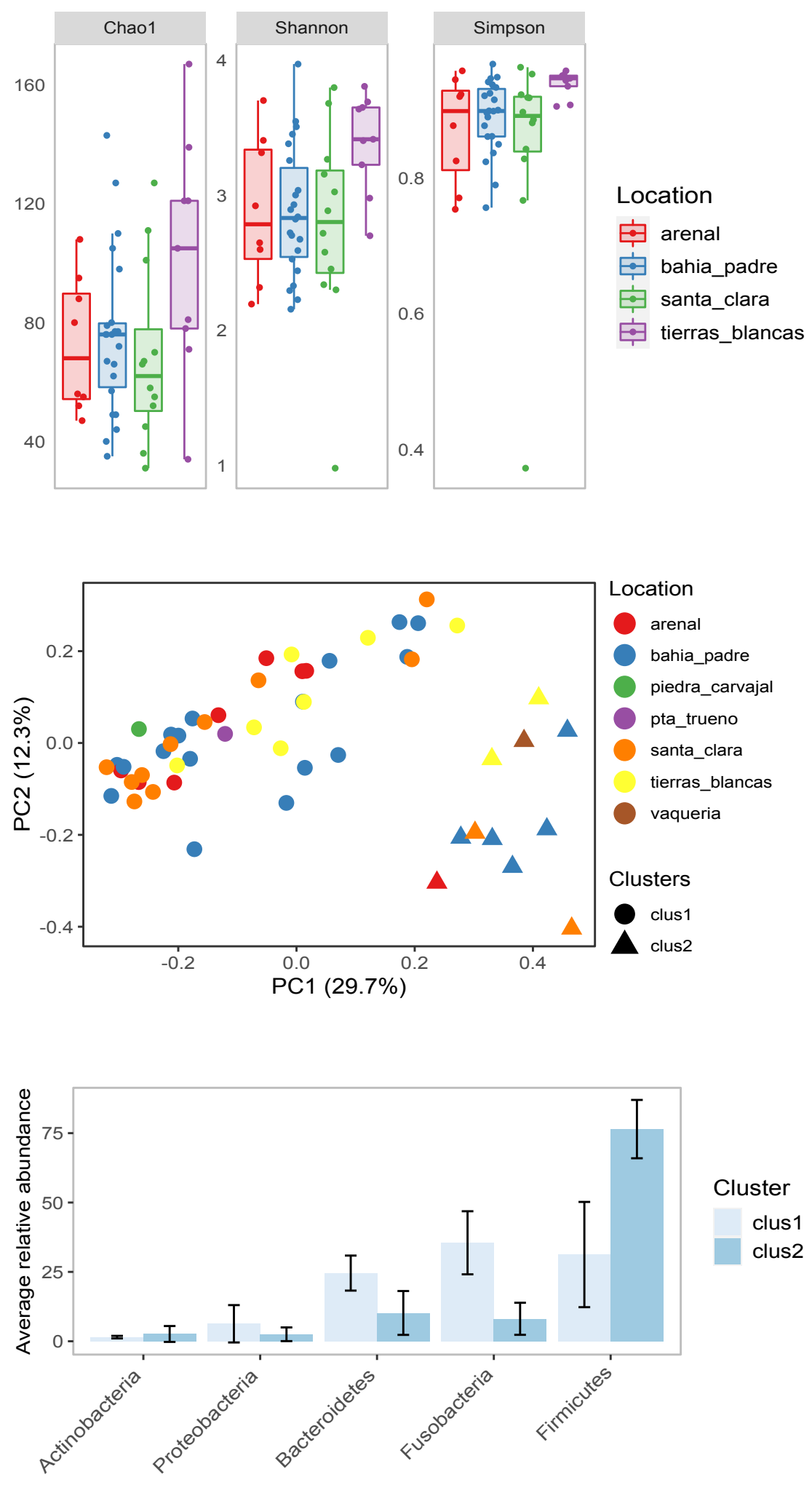


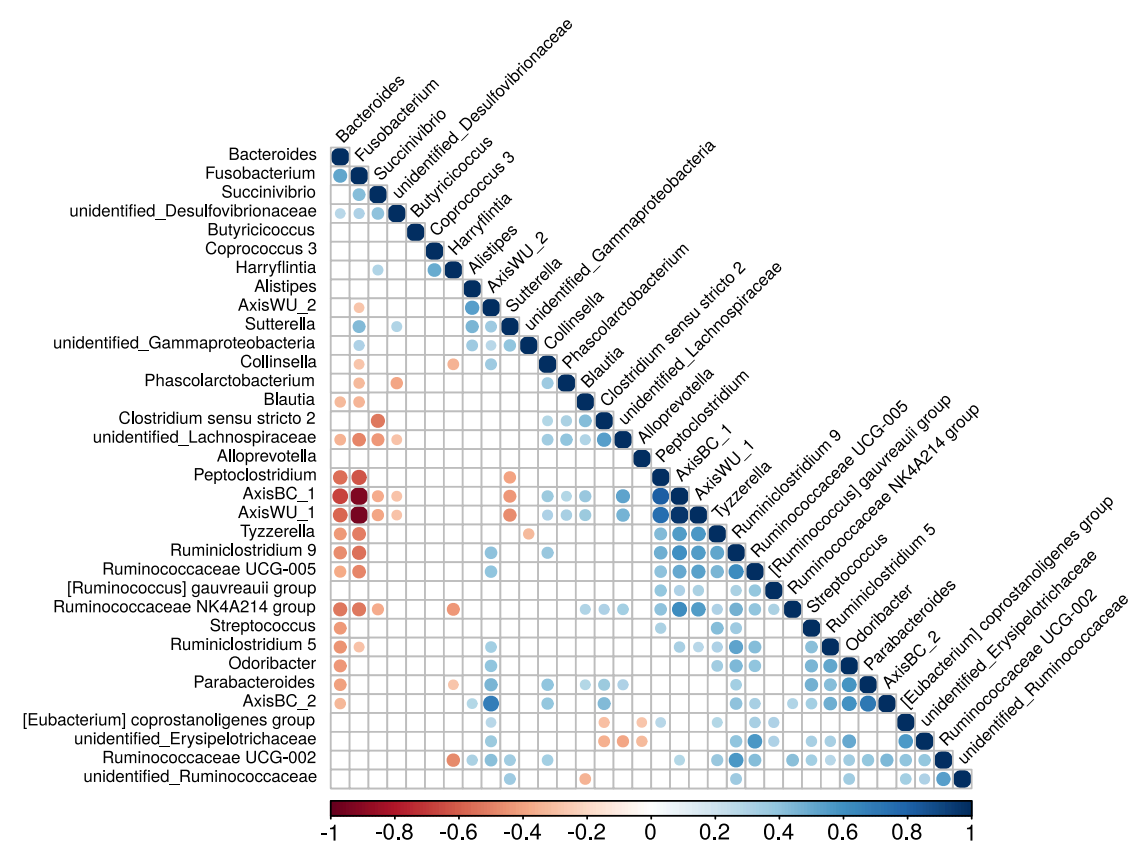

